\title{
The Importance of Ground-Based Radar Observations for Planetary Exploration
}

A white paper submitted to the Planetary Science and Astrobiology Decadal Survey 2023-2032

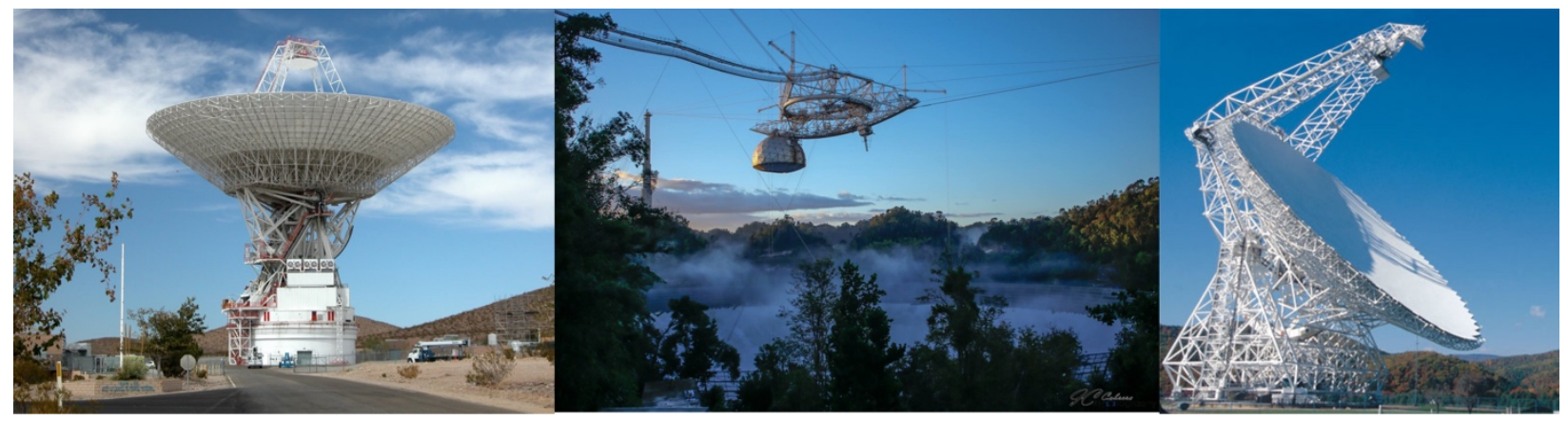

Image Credits: (left) Hal Janzen, (center) Israel Cabrera, and (right) National Radio Astronomy Observatory.

Primary Author: Edgard G. Rivera-Valentín, Lunar and Planetary Institute (LPI), Universities Space Research Association (USRA), (rivera-valentin@lpi.usra.edu)

Co-Authors: Patrick A. Taylor ${ }^{1}$, Carolina Rodriguez Sanchez-Vahamonde ${ }^{1}$, Dylan Hickson ${ }^{2}$, Catherine Neish ${ }^{3,4}$, Marina Brozović ${ }^{5}$, Jennifer Whitten ${ }^{6}$, Heather Meyer ${ }^{7}$, Michael Busch ${ }^{8}$, Bruce A. Campbell ${ }^{9}$, Donald B. Campbell ${ }^{10}$, Jean-Luc Margot ${ }^{11}$, Anne Virkki ${ }^{2}$, Flaviane Venditti ${ }^{2}$, Michael C. Nolan ${ }^{12}$, Ellen S. Howell ${ }^{12}$, Maria Womack ${ }^{2,13}$, and Noemí Pinilla-Alonso ${ }^{2,13}$

${ }^{1}$ Lunar and Planetary Institute, Universities Space Research Association

${ }^{2}$ Arecibo Observatory, University of Central Florida

${ }^{3}$ Department of Earth Sciences, The University of Western Ontario

${ }^{4}$ Planetary Science Institute

${ }^{5}$ Jet Propulsion Laboratory, California Institute of Technology

${ }^{6}$ School of Science and Engineering, Tulane University

${ }^{7}$ Applied Physics Laboratory, Johns Hopkins University

${ }^{8}$ SETI Institute

${ }^{9}$ Center for Earth and Planetary Studies, Smithsonian Institution

${ }^{10}$ Department of Astronomy, Cornell University

${ }^{11}$ Department of Earth, Planetary, and Space Sciences, University of California Los Angeles

${ }^{12}$ Lunar and Planetary Laboratory, University of Arizona

${ }^{13}$ Florida Space Institute, University of Central Florida

Endorsers: Over 50 endorsers! Signatures can be found at the following link: https://tinyurl.com/PlanetaryRadarSupport 


\section{Executive Summary}

Ground-based planetary radar observations have enabled and continue to facilitate the exploration of our solar system through characterization of planets and their moons. This includes spacecraft landing-site characterization, e.g., for Viking (Simpson et al., 1978) and most recently InSight (Putzig et al., 2017) at Mars, and improved target astrometry, such as for Europa (Brozović et al., 2020). The power of radar for planetary geology is its ability to sense subsurface features buried beneath regolith, such as cryptomare on the Moon (Campbell \& Hawke, 2005), and surface features obscured by a dense atmosphere, e.g., Venus (Campbell \& Burns, 1980) and Titan (Campbell et al., 2003). New analytical and modeling techniques (e.g., Virkki \& Bhiravarasu, 2019) as well as laboratory-based insights for radar analysis (e.g., Taylor \& Rivera-Valentín, 2019), along with improvements to existing facilities (see white paper Lazio et al., 2020), motivate a renewed interest in radar studies of planetary surfaces. With a broad fleet of spacecraft exploring the solar system, synergies with ground-based radar studies allow for an improved understanding of planetary processes (see white paper Kofman et al., 2020). In the absence of orbital assets, ground-based radars are a uniquely capable way of continued highresolution (few-meter to kilometer scale) studies of planetary surfaces. Fundamentally, we emphasize that ground-based planetary radar infrastructure must continue to be supported (see white paper Taylor et al., 2020), including further upgrades to existing facilities. Here, we discuss several community-identified priority science questions that ground-based radar studies are particularly suited to help resolve in the next decade.

\section{Technical Overview}

There are two key facilities with transmitter capabilities for planetary radar observations: the 305-m Arecibo Observatory in Puerto Rico and the Goldstone Solar System Radar (GSSR) in California. In the latter case, the 70-m DSS-14 and 34-m DSS-13 elements of the Deep Space Network are used for solar system radar observations. The Arecibo planetary radar system includes an S-Band $(2380 \mathrm{MHz}, 12.6 \mathrm{~cm})$ continuous wave radar with a continuous power of 1 $\mathrm{MW}$ and a P-band $(430 \mathrm{MHz}, 70 \mathrm{~cm})$ pulsed radar with a peak power of $2.5 \mathrm{MW}$. The GSSR can transmit at X-Band $(8560 \mathrm{MHz}, 3.5 \mathrm{~cm})$ at a continuous power of $500 \mathrm{~kW}$ from DSS-14; the 80 $\mathrm{kW}, \mathrm{C}$-band $(7190 \mathrm{MHz}, 4.2 \mathrm{~cm}$ ) DSS-13 is primarily used for asteroid and lunar studies. Other radio telescopes, such as the Green Bank Telescope and the Very Large Array, have been used as receiving antennas in bistatic configurations (i.e., where the transmitter and receiver are not colocated). Additionally, the Jicamarca Radio Observatory in Peru, which operates at VHF frequencies (49.9 MHz; $6 \mathrm{~m}$ ), has recently been used for lunar radar experiments (Vierinen et al., 2017). Because radio wave attenuation in solid media is frequency dependent, these radar telescopes are complementary as they sense down to different depths, revealing obscured features, and are also sensitive to different regolith grain sizes.

Typically, ground-based planetary radar observations involve the transmission of circularly polarized light to conduct either continuous wave (CW) experiments or delay-Doppler experiments. The average radar albedo over the total illuminated area in both the same-sense circular (SC) and opposite-sense circular (OC) polarizations is measured with CW experiments. Radar albedo is sensitive to near-surface properties (e.g., composition, density, and porosity); the ratio of the SC and OC albedo gives the circular polarization ratio (CPR), which is sensitive to near-surface, wavelength-scale roughness. Delay-Doppler experiments provide high-resolution (few-meter to kilometer scale), ground-based imaging of planetary surfaces (e.g., Figure 1), 
revealing near-surface geology and variations in regolith properties. Another experiment is radar speckle interferometry, which is used to study the target's spin state. To date, speckle interferometry has been used to study the spin state of Mercury, Venus, Europa, and Ganymede. At Mercury, the data revealed the presence of a liquid core and enabled a measurement of its size (Margot et al., 2007; 2012; 2018), results confirmed by MESSENGER.
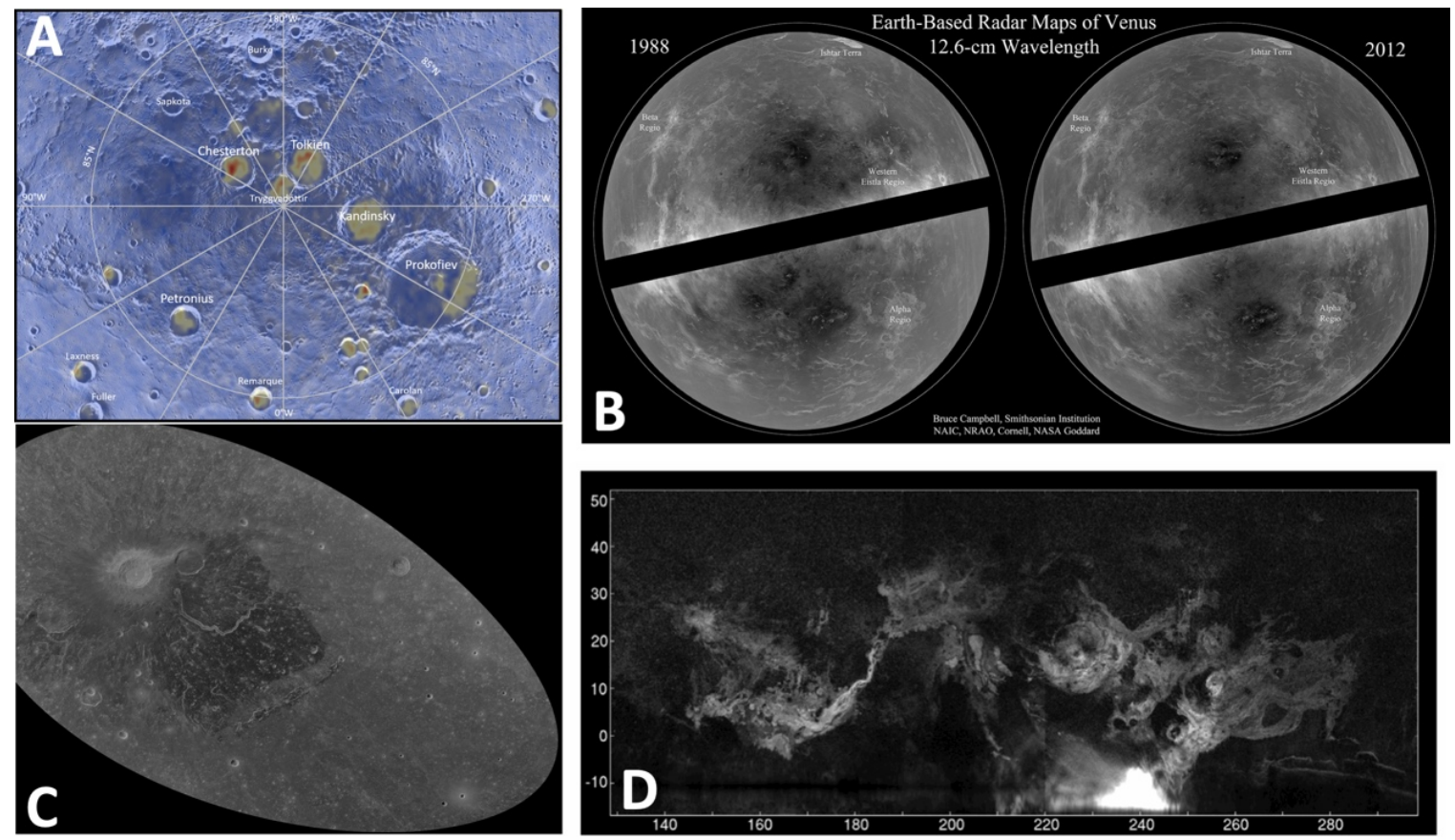

Figure 1: Mosaic of radar-derived products. (A) Total radar backscatter (in color) overlain onto MESSENGER MDIS data of the north pole revealing ice deposits (Rivera-Valentín et al., 2020), (B) Venus radar maps from 1988 and 2012 that are part of the long-term observing campaign to identify volcanic activity on Venus, (C) SC radar image of Aristarchus Plateau used to constrain the properties of the ancient pyroclastic event (Campbell et al., 2008), (D) SC radar image of Mars demonstrating the surface obscured connection between ancient volcanic provinces (Harmon et al., 2012).

\section{Planetary Radar Observations in the Next Decade}

Although ground-based radar facilities are primarily used for small body astrometry and characterization particularly for planetary defense (e.g., see white papers Mainzer et al., 2020; Virkki et al., 2020), detailed radar imaging and analyses of large bodies have resulted in profound revelations about their surface histories and interior structure. Polarimetric radar studies can constrain regolith grain size and shape distribution, bulk density, composition, and roughness, and can also be used to infer the presence of buried ice. Here we review earlier radar findings and discuss priority science questions that in the next decade can be elucidated with groundbased radar observations. To guide future observations, in Table 1 we show planetary observing opportunities with the Arecibo Observatory in the next decade.

Table 1: Arecibo observing opportunities from 2023- 2032. The Moon is only observable in bistatic configuration.

\begin{tabular}{|r|c|c|}
\hline Planet & Years Observable from Arecibo & Best Year \\
\hline Mercury & Yearly & 2028 \\
\hline Venus & $2023,2025,2028,2031$ & 2025 \\
\hline Moon & Monthly & NA \\
\hline Mars & $2025,2027,2029$ & 2025 \\
\hline Jupiter & $2023-2028$ & 2023 \\
\hline Saturn & $2026-2032$ & 2032 \\
\hline
\end{tabular}




\subsection{Mercury}

Arecibo radar observations revealed the prominence and variety of fresh impact craters and their ray systems on Mercury (Harmon et al., 2007), yet a lot more remains to be done in characterizing the planet's smooth plains, volcanic deposits, and intercrater plains, including their compositions (radar loss tangents), relationship with impact structures (e.g., Rivera-Valentín \& Barr, 2014), and emplacement histories. The wealth of geological and compositional information provided by MESSENGER now provides excellent context that can help better decipher radar products. Indeed, continued radar observations are complementary to spacecraft data and can facilitate future landed missions (e.g., white paper Byrne et al., 2020). The most notable radar discovery at Mercury was the identification of radar-bright features at its poles (e.g., Slade et al., 1992; Harmon \& Slade, 1992), which scattering models suggested were due to pure ice (Butler et al., 1993). The MESSENGER spacecraft confirmed that the radar-bright features are associated with locations persistently in shadow (Chabot et al., 2013) that are on average hydrogen-rich (Lawrence et al., 2013), indicative of water ice. Interestingly, though, MESSENGER revealed that many permanently shadowed regions in the North Pole lack radarbright deposits (Deutsch et al., 2016). Possible explanations include: (1) incomplete longitudinal coverage by radar measurements, (2) a lack of water ice, which implies that not all cold-traps are occupied, (3) thin water ice deposits that do not cause a significant radar return, or (4) water ice buried below the radar penetration depth, which could imply an ancient deposit. Improved radar analysis techniques applied to new Arecibo observations combined with MESSENGER data can help constrain these possibilities (e.g., Rivera-Valentín et al., 2020). Thus, a Mercury Exploration Assessment Group priority science question (see white paper Deutsch \& Chabot, 2020) that radar observations would help to address is:

Science Question: What are the origin, age, \& characteristics of the Mercurian polar deposits?

\subsection{Venus}

Ground-based radar studies of Venus provided our early understanding of its surface geology, such as the paucity of impact craters and the abundance of volcanic landforms. They have also provided coverage of large regions in two senses of circular polarization, which are sensitive to different scales of surface roughness. For example, Arecibo radar observations are more sensitive to fine-grained deposits associated with crater ejecta deposits and their full extent can be more readily identified, in comparison with the Magellan SAR data (Carter et al., 2004; Campbell et al., 2015). Another application has been to measure variations in the average rotation rate of Venus using long-term Earth-based observations (Campbell et al., 2019), which have implications for selecting future landing sites, as well as length-of-day variations using radar interferometry (Margot et al., 2019). Other studies that can benefit from the long-term record are surface change detection. Many regions of recent or active volcanic activity have been proposed, with several occurring on the timescale of Earth-based surface observations (e.g., Bondarenko et al., 2010; Shalygin et al., 2015; Campbell et al., 2017). Indeed, using laboratory-constrained weathering rates for Venusian lava flows, Filiberto et al. (2020) suggested that some flows may be no more than a few years old. While many remote observations have suggested ongoing volcanic activity, there has yet to be an observation of surface change. The Venus Exploration Analysis Group identified a science question (see white paper Smrekar et al., 2020) that continued radar observations can address:

Science Question: What is the volcanic history of Venus and is it presently volcanically active? 


\subsection{The Moon}

On the Moon, radar studies have revealed extensive, complex, and buried volcanic deposits, and have characterized both impact melt and crater ejecta (e.g., Thompson et al., 1981; Neish et al., 2013; 2014; Campbell, 2016). In particular, Arecibo P-band images significantly extended the inventory of mare basalts on the Moon's nearside (e.g., Campbell \& Hawke, 2005). Indeed, at least $12 \%$ of mare basalts are buried (Whitten and Head, 2015) and are only visible by either impact excavation of basaltic material or radar observations. In the absence of a P-band instrument in orbit, improvements to Arecibo would provide higher spatial resolution P-band observations (e.g., Campbell et al., 2019). Additionally, ground-based VHF radar observations provide a new avenue for exploring lunar geology by probing to greater depths (e.g., Vierinen et al., 2017). Since the arrival at the Moon of orbital radars, ground-based telescopes have provided complementary information and a means of taking bistatic observations. The LRO's Mini-RF instrument has made extensive use of bistatic observations, transmitting from Arecibo or GSSR and receiving with Mini-RF. Bistatic observations allow for the characterization of the nearsurface radar scattering properties as a function of bistatic angle, which eliminates some ambiguities associated with monostatic observations. Additionally, they can be used to search for a coherent backscatter opposition effect indicative of thick layers of subsurface water ice (e.g., Patterson et al., 2017), which monostatic Arecibo observations were not able to detect (Stacy et. al., 1997). The availability of ground-based radar assets as transmitters will be critical for continued bistatic observations during the LRO extended mission. With the renewed effort to return humans to the Moon, an open question ground-based radar assets can address is:

Science Question: What are the characteristics and volatile inventory of lunar landing sites?

\subsection{Mars}

Beyond landing-site characterization, ground-based radars can provide another avenue to help resolve challenges in interpreting orbital datasets. Anomalous radar properties have been identified throughout the south polar layered deposits (SPLD) using spacecraft instruments (e.g., Whitten \& Campbell, 2018). A robust measurement of the surface scattering properties with Arecibo can provide important information about the surface roughness across the SPLD at a scale that is not attainable with current datasets. Thus, radar can be used to enhance the interpretation of orbital measurements. Additionally, ground-based radar imaging of Mars has helped reveal its volcanic history. Using Arecibo radar data, Harmon et al. (2012) identified connections between ancient volcanic features obscured by dust. The high CPRs measured for lava flows in the volcanic provinces of Tharsis, Elysium, and Amazonis suggest the material is extremely blocky or highly disrupted. This texture is uncommon on Earth, where it is produced by terrestrial andesitic, blocky lava flows, such as those seen in the SP Flow in Arizona (Campbell, 2012) and in Craters of the Moon National Monument and Preserve in Idaho (Neish et al., 2017). However, recent work examining the meter-scale roughness properties of Martian lava flows using high-resolution optical data sets revealed they are relatively smooth (Rodriguez SanchezVahamonde \& Neish, 2020). Consequently, to better understand volcanism on Mars, it is necessary to study roughness at different scales. Radar roughness data is thus complementary to the orbital measurements in understanding the emplacement mechanisms of these lava flows. An open question that continued Arecibo radar observations would be invaluable at resolving is: Science Question: What are the physical characteristics of Martian lava flows, and what does this tell us about their emplacement history? 


\subsection{Galilean Moons}

Past radar observations of the Galilean moons obtained echo power spectra in order to (1) determine their scattering properties (Ostro, 1992; Ostro et al., 1993), (2) acquire high-resolution (few tens of $\mathrm{km}$ ) delay-Doppler images and radar reflectance maps in order to reveal details of their surfaces (Harcke et al., 2001), and (3) collect high-precision (few km) ranging measurements in order to improve their ephemerides (Harmon et al., 1994; Brozović et al., 2020). Long-term radar ranging to the Galilean moons will maintain high-fidelity satellite ephemerides, which is important for upcoming missions, such as NASA's Europa Clipper. Additionally, ongoing radar speckle interferometry of Europa and Ganymede will provide constraints on their obliquities and moments of inertia (Margot et al., 2013), which helps resolve their internal structure. Continued radar observations are particularly important because they reveal tidal interactions in the jovian system that are responsible for, e.g., the volcanic activity on lo and Europa's subsurface ocean. Tidal dissipation is parameterized by $k_{2} / Q$, where $k_{2}$ is the Love number and $Q$ is the quality factor. NASA's Juno mission recently accurately measured $k_{2}$ (Durante et al., 2020). This result combined with $k_{2} / Q$, which can be estimated from an orbital fit, can be used to determine Jupiter's quality factor $Q$, and thus provide strong constraints on its interior. Lainey et al. (2009) obtained a nominal value of $k_{2} / Q$ by fitting orbits to astrometry from mutual events, occultations and eclipses, but these measurements were more than an order of magnitude less precise than the radar ranging measurements (Harmon et al., 1994; Brozović et al., 2020). Hence, groundbased radar observations can provide further refinement of the $k_{2} / Q$ value; thus, answering: Science Question: What is the internal structure of Jupiter?

\subsection{Titan}

Prior to the Cassini mission, ground-based radar observations had found evidence for specular reflections near Titan's equator, indicating large surfaces smooth at the wavelengthscale (Campbell et al., 2003); however, no obvious equatorial lakes were observed by Cassini, which found lakes concentrated exclusively near the poles. Thus, these observations may be indicative of smooth surfaces saturated in liquid hydrocarbons (Black et al., 2011) or smooth sedimentary deposits in paleolakes (Hofgartner et al., 2020). Further radar observations could help resolve this. The Cassini mission ended in 2017; the future exploration of Titan will now be up to the recently selected NASA Dragonfly mission. The Cassini RADAR instrument provided the highest resolution global views of Titan's surface, enabling the Dragonfly team to identify landing sites and plan traverses on a larger scale than is visible from the rotorcraft lander. Cassini RADAR also provided the only topography data available for Titan, through radar stereogrammetry, altimetry, and the innovative SARTopo technique (Stiles et al., 2009). Ground-based radar observations of Titan will be possible in the late 2020s (see Table 1). Precise radar astrometry of Titan in the next decade could provide constraints on Titan's orbital drift rate (Lainey et al., 2020). Additionally, these observations will provide information on how the surface may have changed since the end of the Cassini mission to the scheduled landing of Dragonfly in 2034, enabling improved preparation for the mission. In the absence of active missions in the Saturn system, upcoming radar observations can further our understanding of Titan and prepare for Dragonfly by answering an Outer Planets Assessment Group Titan exploration goal ${ }^{1}$ : Science Question: What processes are active on Titan's surface and how have these processes, and the surface of Titan, changed over time?

${ }^{1}$ https://www.Ipi.usra.edu/opag/goals-08-28-19.pdf 


\section{Findings \& Recommendations}

Ground-based planetary radar observations pave the way for spacecraft exploration of the solar system, and provide complementary data to orbital assets. They also allow for higher-order analysis and understanding of planetary surfaces and interiors well beyond the end of spacecraft missions. In the absence of orbital assets, ground-based radar observations are a uniquely capable and cost-efficient way of continued studies of planetary surfaces. Such data is invaluable for future landed missions (e.g., Byrne et al., 2020). Although much has been accomplished previously with radar observations of planetary bodies, potential and upcoming upgrades to facilities (e.g., Lazio et al., 2020), as well as improved data analysis techniques (e.g., Virkki \& Bhiravarasu, 2019), insights from laboratory experiments (e.g., Taylor \& Rivera-Valentín, 2019), and synergies with recent and upcoming missions (e.g., Kofman et al., 2020; Rivera-Valentín et al., 2020) motivate a renewed interest in radar studies of planetary surfaces. The importance of ground-based radar facilities for planetary exploration coupled with their importance for small body astrometry and characterization for planetary defense purposes (e.g., Mainzer et al., 2020; Taylor et al., 2020; Virkki et al., 2020) furthers the value of these observatories.

For the upcoming decade we present the following major findings and recommendations:

\section{Findings:}

- Ground-based planetary radar observations of the solar system coupled with radar scattering models are invaluable in answering high priority planetary science questions in the coming decades.

- Continued ground-based radar observations provide opportunities to detect changes on planetary surfaces, placing new constraints on present-day processes (e.g., volcanism on Venus and lakes on Titan) and to measure moments of inertia (e.g., Venus).

- Ground-based radar products (e.g., radar albedo, roughness) are complementary to orbital datasets, offering new opportunities to improve interpretation of each dataset, as well as higher order analysis.

- Ground-based radar enables robotic and human exploration of the solar system through landing site characterization, including identification of hazards and volatile reservoirs for in-situ resource utilization, and ultra-precise astrometric measurements.

\section{Recommendations:}

- Fundamentally, we emphasize that ground-based planetary radar infrastructure must continue to be supported, including investment in continued maintenance and upkeep of existing facilities, as well as further upgrades. This recommendation is further detailed in the white paper by Taylor et al. (2020) on access to ground-based radar facilities.

- Investment in continued ground-based radar observations in the next decade is needed in order to facilitate identification of present-day activity across the solar system, e.g., on Venus where it can shed light on volcanic activity, and on Titan where it can assist in observing strategies for NASA's Dragonfly.

- Investment in laboratory measurements of radar scattering properties of analog material under realistic conditions and over a range of bistatic angles is needed in order to improve interpretation of radar data. Additionally, investment in complementary field measurements is also needed to elucidate surface processes, such as volcanism and impact cratering. Such laboratory and field results are needed to help refine radar scattering models and their application to natural surfaces. 


\section{Statement on Diversity, Equity, and Inclusion}

Studying planetary processes with radar requires drawing on different perspectives. Thus, it is critical that the community fosters an interdisciplinary, diverse, equitable, inclusive, and accessible environment. We strongly encourage the decadal survey to closely consider the state of the profession and issues of equity, diversity, inclusion, and accessibility. Information on the demographics of the field along with recommendations can be found in e.g., white papers Milazzo et al. (2020), Piatek et al. (2020), Rivera-Valentín et al. (2020), and Rathbun et al. (2020).

\section{References}

Black, G. J. et al. (2011) Icarus 212, 300-320.

Bondarenko, N. V. et al. (2010) GRL 37, L23202.

Brozović, M. et al. (2020) Astronomical Journal 159, 149.

Butler, B.J. et al. (1993) JGR 98, 15003-21.

Campbell \& Hawke (2005) JGR-P 110, E09002.

Campbell, B. A. et al. (2008) Geology 36, 135-138.

Campbell, B. A. (2012) JGR 117, E06008.

Campbell, B. A. et al. (2015) Icarus 250, 123-130.

Campbell, B. A. et al. (2016) Pub. Astron. Soc. Pacific 128, 062001.

Campbell, B. A. et al. (2017) JGR 122, 1580-1596.

Campbell, B. A. et al. (2019) Icarus 332, 19-23.

Campbell \& Burns (1980) JGR-SP 85, 8271-8281.

Campbell, D. B. et al. (2003) Science 302, 431-434.

Carter, L. M. et al. (2004) JGR 109, E06009.

Chabot, N. L. et al. (2013) JGR-P 118, 26-36.

Deutsch, A. N. et al. (2016) Icarus 280, 158-171.

Durante, D. et al. (2020) GRL 47, e2019GL086572.

Filiberto, J. et al. (2020) Science Advances 6, eaax7445.

Harcke, L. J. et al. (2001) LPSC, Abstract\# 1369.

Harmon \& Slade (1992) Science 258, 640-643.

Harmon, J. K. et al. (1994) AJ, 107, 1175-1181.

Harmon, J.K. et al. (2007) Icarus 187, 374-405.

Harmon, J.K. et al. (2012) Icarus 220, 990-1030.

Hofgartner, J. D. et al. (2020) Nature Comm. 11, 2829.

Lainey, V. et al. (2009) Nature 459, 957-959.

Lainey, V. et al. (2020) Nat. Astro, doi:

10.1038/s41550-020-1120-5.

Lawrence, D. J. et al. (2013) Science 339, 292-296.

Margot, J. L. et al. (2007) Science 316, 710-714.

Margot, J. L. et al. (2012) JGR 117, E00L09

Margot, J. L. et al. (2013) AAS 221, id 314.04.

Margot, J. L. et al. (2018) Mercury - The View after

MESSENGER, Cambridge University Press, 85-113.

Margot, J. L. et al. (2019) DPS, Abstract \#412.

Neish, C. D. et al. (2013) JGR-P 118, 2247-2261.

Neish, C. D. et al. (2014) Icarus 239, 105-117.

Neish, C. D. et al. (2017) Icarus 281, 73-89.

Ostro, S. J. et al. (1992) JGR 97, 18277-18244.

Ostro, S. J. et al. (1993) Reviews of Modern Physics 65, 1235-1279.
Patterson, G.W. et al. (2017) Icarus 283, 2-19.

Putzig, N. E. et al. (2017) Space Sci. Rev. 211, 135-146.

Rivera-Valentin \& Barr (2014) EPSL 391, 234-242.

Rivera-Valentín et al. (2020) LPSC, Abstract \#1593.

Rodriguez Sanchez-Vahamonde, C. \& Neish, C. D.

(2020) Planetary Science Journal, under review.

Shalygin, E. V. et al. (2015) GRL 42, 4762-4769.

Simpson, R. A. et al. (1978) Icarus 36, 153-173.

Slade, M. A. et al. (1992) Science 258, 635-640.

Stacey, N. J. S. et al. (1997) Science 276, 1527-1530.

Stiles, B. W. et al. (2009) Icarus 202, 584-598.

Taylor \& Rivera-Valentín (2019) Orbital Debris Conf., Abstract\# 2109.

Thompson, T. W. et al. (1981) Icarus 46, 201-225.

Vierinen, J. et al. (2017) Icarus 297, 179-188.

Virkki \& Bhiravarasu (2019) JGR-P 124, 3025-3040.

Whitten, J. L. \& Head, J. W. (2015) Icarus 247, 150-171.

Whitten, J. L. \& Campbell, B. A. (2018) JGR-P 123, 1541-1554.

Cited White Papers

Byrne et al. (2020) "On the Case for Landed Mercury Science"

Deutsch \& Chabot (2020) "Science Opportunities offered by Mercury's Ice-Bearing Polar Deposits"

Kofman et al. (2020) "Synergies between groundbased and space-based observations ..."

Lazio et al. (2020) "The next-generation planetary radar"

Mainzer et al. (2020) "The future of planetary defense in the era of advanced surveys: ..."

Milazzo et al. (2020) "DEIA 101: ..."

Piatek et al. (2020) "Accessibility within planetary science"

Smrekar et al. (2020) "Habitability, geodynamics, and the case for Venus"

Rathbun et al. (2020) "Who is missing in planetary science: ... part II"

Rivera-Valentín et al. (2020) "Who is missing in planetary science: ... part 1"

Taylor et al. (2020) "Ground-based radar for planetary science and planetary defense"

Virkki et al. (2020) "Near-Earth object characterization using ground-based radar systems" 